\title{
Linoleic Acid-A Feasible Preventive Approach for Visceral Leishmaniasis
}

\author{
Sheetal Saini and Ambak Kumar Rai* \\ Department of Biotechnology, Motilal Nehru National Institute of Technology Allahabad, Prayagraj, India
}

Keywords: visceral leishmaniasis, malnutrition, $\omega-6$ polyunsaturated fatty acid, linoleic acid, protective immunity

\section{INTRODUCTION}

Kala-azar, also known as visceral leishmaniasis (VL), remains one of the top parasitic diseases and is fatal if left untreated in over $95 \%$ of cases. The condition is characterized by irregular fever bouts, weight loss, hepato-splenomegaly (enlargement of the spleen and liver) and anemia. Along with Sudan, Ethiopia, and Brazil, the Indian subcontinent contributes to $~ 90 \%$ of the global burden of visceral leishmaniasis (VL) (1). Post kala-azar dermal leishmaniasis (PKDL), a clinical complication of VL, where infection reoccurs in the dermal region after successful treatment, is also a challenge. The presence of the Leishmania parasite in the cutaneous region may serve the

OPEN ACCESS

Edited by:

Julio Villena,

CONICET Centro de Referencia para Lactobacilos (CERELA), Argentina

Reviewed by:

Susana Salva,

CERELA-CONICET, Argentina Paulraj Kanmani,

Tohoku University, Japan

*Correspondence:

Ambak Kumar Rai ambakrai@mnnit.ac.in

Specialty section:

This article was submitted to Nutritional Immunology, a section of the journal

Frontiers in Nutrition

Received: 03 January 2021 Accepted: 09 February 2021 Published: 26 February 2021

Citation:

Saini S and Rai AK (2021) Linoleic

Acid-A Feasible Preventive Approach for Visceral Leishmaniasis.

Front. Nutr. 8:649025

doi: 10.3389/fnut.2021.649025 purpose of a reservoir for the consistent dissemination of disease. Therapeutic vaccines and newer potential drugs are under development/research, but unfortunately, we have nothing in hand. Thus, there is still a lot that needs to be done to achieve the absolute eradication of the disease. Prevalent malnutrition and resultant inadequate immune response are the practical limitations restricting the elimination of VL from endemic regions. Consequently, there is a need to look for newer methods to condition the immune system for a protective cellular response against Leishmania infection in endemic areas as a preventive measure.

Malnutrition is always considered as a possible risk factor for the advancement of VL $(2,3)$. It is associated with reduced immune response and increased visceralization of the parasite (4). Linoleic acid (LA) is an essential fatty acid (EFA) for humans due to the unavailability of fatty acid desaturase (FAD)-12. This enzyme is present in plants and thus, for humans, plant-derived diets are the primary source of LA. Arachidonic acid (AA, $\omega-6)$ is the derivative of LA and is known to set a background for instant innate immune response with its metabolites, i.e., eicosanoids. Humans can acquire arachidonic acid (AA, $\omega-6)$ either from animal based-diet or FAD-6 and FAD-5 synthesize AA from dietary LA (5). The low levels of LA are also reported in individuals suffering from malnutrition $(6,7)$. The inadequate dietary supply of LA is very much possible in endemic areas. We have also found low levels of LA in VL patients' serum compared to healthy individuals (8). Furthermore, it is established that the deficiency of EFA (i.e., LA) causes loss of water from the skin resulting in dark and patchy skin, which are the hallmark symptoms of VL disease $(9,10)$. This could be one of the plausible reasoning to explain skin darkening in VL patients. Malnutrition is reported to lead a relative increase in anti-inflammatory eicosanoids as compared to pro-inflammatory eicosanoids, which contributes to the compromised innate immune response against Leishmania infection (11).

Abbreviations: VL, Visceral Leishmaniasis; LA, Linoleic acid; Ld, Leishmania donovani; AA, Arachidonic acid; PUFA, Polyunsaturated fatty acids; EFA, Essential fatty acid; 5-LO, 5-lipoxygenase; LTB4, Leukotriene B2; PGE2, Prostaglandin E2; Macrophages, $\mathrm{M} \phi ;$ LdMv, Leishmania donovani. derived microvesicles. 


\section{LEISHMANIA INFECTION AND $\omega-6$ POLYUNSATURATED FATTY ACIDS (PUFA)}

The pathogenesis of VL significantly depends upon macrophage $(\mathrm{m} \phi)$-Leishmania interactions and further their encounter with T cells. As major components of the cellular membrane, PUFA plays a pivotal role in maintaining the membrane fluidity, which is essential for appropriate antigen presentation to $\mathrm{T}$ cells and modulate inflammatory and immune responses
(12). Classically, human immune/inflammatory cells ( $\mathrm{m} \phi$, neutrophils, mast cells, etc.) are high in $\omega-6$ PUFA compared to $\omega-3$ (13). LA is converted to AA and stored in the plasma membrane of the cells, especially immune cells like tissue $\mathrm{m} \phi$, dendritic cells, and neutrophils (14). Precisely, AA is positioned at the 2-acyl motif of membrane phospholipids (mainly phosphatidylcholine, phosphatidylethanolamine and phosphatidylinositol). M $\phi$ enriched with PUFAs, especially AA ( $\omega-6)$ showed $~ 50 \%$ enhancement of phagocytic and adhesion

A

Linoleic acid and visceral leishmaniasis

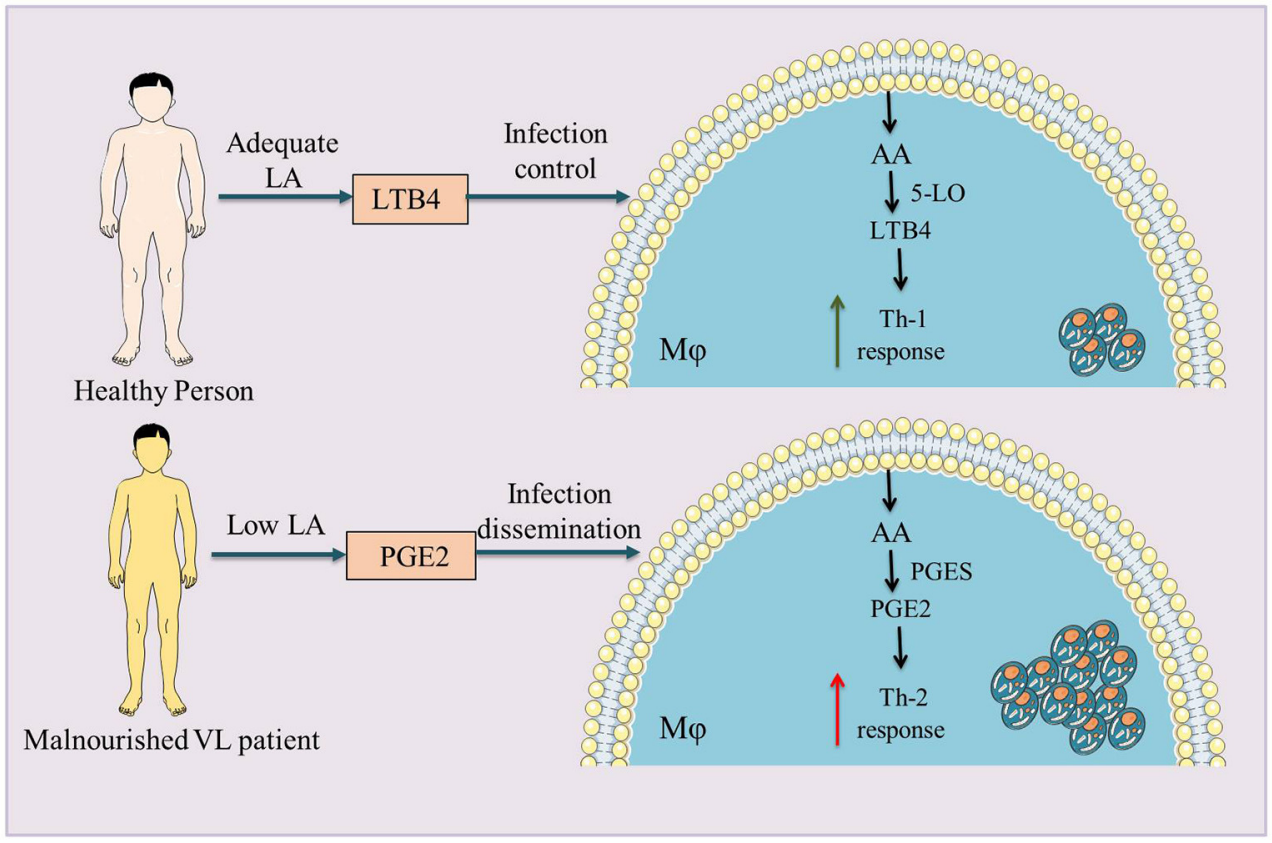

B

Dual way action of linoleic acid against Leishmania donovani

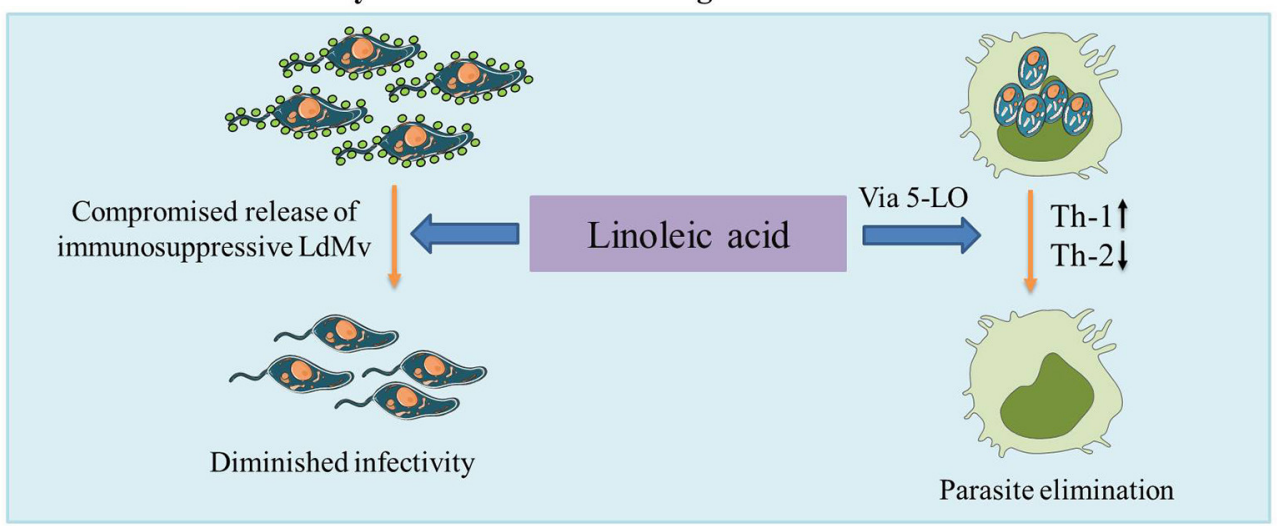

FIGURE 1 | The role of linoleic acid against visceral leishmaniasis (VL) infection. (A) Malnutrition is associated with VL and in malnourished individuals as well as VL patients, low levels of linoleic acid (LA) are observed $(7,8)$. Malnutrition leads to a relative increase in anti-inflammatory prostaglandin E2 (PGE2) compared to pro-inflammatory leukotriene B4 (LTB4) (11). Higher levels of PGE2 with lower levels of LTB4 create an immunosuppressive environment (upregulated Th2 response) in the host, which leads to the advancement of $\mathrm{VL}$ infection. (B) Linoleic acid plays a dual way protective role against Leishmania donovani infection (i) by inhibiting the release of Leishmania donovani derived microvesicles (LdMV) from promastigote form of the parasite (36) and (ii) by promoting the protective Th-1 type pro-inflammatory (via the 5-lipoxygenase (5-LO) pathway and suppressing the Th-2 type anti-inflammatory immune response (8). LA, linoleic acid; LTB4, leukotriene B4; AA, arachidonic acid; 5-LO, 5-lipoxygenase; M申, macrophage; VL, visceral leishmaniasis; PGE2, prostaglandin E2; PGES, prostaglandin E2 synthases; LdMv, Leishmania donovani derived microvesicles. 
capacity (15). LA also evokes superoxide release from neutrophils and $\mathrm{m} \phi$ (16), which is essential for eliminating the Leishmania parasite (17). LA is the precursor of $\omega-6$ PUFAs in mammals and their deficiency at the cellular level impairs cell-to-cell interaction by modifying cell adhesion (18). This deficiency could cause improper immunological synapse formation, leading to compromised antigen presentation by antigen-presenting cells and inadequate lymphocyte activation (19), which may disseminate the disease.

AA viz. derivative of LA is converted to various metabolites belonging to either anti-inflammatory or pro-inflammatory classes, collectively called eicosanoids due to a 20-carbon (eicosa) precursor. These are the critical factors in inflammation and modulate the immunopathogenesis of various infections and auto-immune disorders (20). Among all eicosanoids, the effect of prostaglandin E2 (PGE2) and leukotriene B4 (LTB4) have been studied most widely. Immunologically, prostaglandins (PGs) are considered as anti-inflammatory and leukotrienes (LTs) as pro-inflammatory. PGE2 suppresses immune cells' proliferation, inhibits NK cell activity, upregulates IL-10 release by $\operatorname{m} \phi(21,22)$ and reduces the production of Th1 cytokines (IFN- $\gamma$, IL-2, TNF$\alpha$, IL-1, and IL-6) by Th1 cells (23). On the other hand, LTB4 increases vascular permeability, enhances ROS generation and promotes NK cell activity (16). LTB4 enhances pro-inflammatory mediators (TNF, IL-1, IL-6, IL-2, IL-12, IFN- $\gamma$, and iNOS) and loss of 5-lipoxygenase (LTB4 final product) leads to decreased phagocytosis of by $\mathrm{m} \phi$ and neutrophils $(24,25)$.

During leishmaniasis, the Leishmania parasite induces PGE2 generation in host $m \phi$ and aid parasite survival $(26,27) . L$. donovani exploits the PGE2/EP2 pathway to reduce protective cytokines (TNF- $\alpha$ and IL-17) (28) and, along with arginase-I and TGF- $\beta$, is known to create a favorable immunosuppressive microenvironment for L. amazonensis dissemination (29). Sandfly (L. longipalpis) saliva is known to create a suppressive atmosphere in $\mathrm{m} \phi$ by inducing PGE2 production and favors L. infantum infection $(30,31)$. On the other hand, LTB4 formation is required for L. amazonensis elimination from $\mathrm{m} \phi$ (32). LTB4 via BLT1 receptor increases ROS generation and potentiates $\mathrm{m} \phi$ leishmanicidal activity. The decreased expression of the BLT1 receptor after Leishmania infection is indicative of a parasite escape mechanism for the chronic and sustained disease (33). Among other experimental models, the Syrian golden hamster (Mesocricetus auratus) is regarded as the best to investigate the immunopathogenesis in $\mathrm{VL}(34,35)$. We have demonstrated the organ-specific role of LTB4 and PGE2 in experimental VL (L. donovani infected M. auratus). 5-

\section{REFERENCES}

1. Burza S, Simon L, Croft Marleen B. Leishmaniasis. Lancet. (2018) 392:95170. doi: 10.1016/S0140-6736(18)31204-2

2. Harrison LH, Naidu TG, Drew SJ, de Alencar JE, Pearson RD. Reciprocal relationships between undernutrition and the parasitic disease visceral leishmaniasis. Rev Infect Dis. (1986) 8:447-53. doi: 10.1093/clinids/ 8.3.447 lipoxygenase (final product LTB4) was prominent in the liver, which contained the parasitic load and the spleen showed upregulated expression of PGE2 synthases (final product PGE2) along with uncontrolled parasite burden (25). The dietary precursor of these eicosanoids, i.e., LA showed a protective response against VL infection in pre-clinical studies. LA inhibited the Th-2 response and promoted Th-1 response, resulting in significantly low $L$. donovani infection in $m \phi$ (8). LA also reduced the release of immunosuppressive extracellular vesicles (microvesicles specifically) from $L$. donovani parasite (36). Taken together, LA plays a dual-way protective role in the immune response against $L$. donovani infection, firstly by inhibiting the release of $L d \mathrm{Mv}$ and secondly promoting the Th-1 type immune response via the 5-LO pathway (Figure 1).

\section{DISCUSSION}

Despite the established protective role of LBT4 in VL, the possibilities of their therapeutic applications are limited due to their transient nature and cost issues. Thus, instead of using eicosanoids, LA, their dietary precursor, may have a beneficial role in disease containment. Higher levels of LTB4 but not PGE2 have been observed in the serum of LA supplemented healthy individuals (37). Varying concentrations of LA are already present in edible oils (Safflower $>75 \%$; Sunflower $>60 \%$; Soybean $>50 \%$; Sesame $>40 \%$, Rice bran $>30 \%$; Groundnut $>$ $25 \%$; Peanut $>15 \%$, Mustard $>10 \%$, Olive $\sim 10 \%$ and Coconut $\sim 1 \%)$. There are no known side effects of LA supplementation in humans. On the contrary, LA intake is inversely associated with coronary heart disease risk. Summarily, a shift in dietary habits from LA-poor oils to LA-rich oils (safflower, sunflower, sesame, etc.) may have beneficial effects on disease containment in endemic areas.

\section{AUTHOR CONTRIBUTIONS}

SS drafted and wrote the manuscript. AR critically revised and edited the manuscript for important intellectual content. Both authors contributed to the article and approved the submitted version.

\section{ACKNOWLEDGMENTS}

Council of Scientific and Industrial Research, Government of India for providing fellowship to SS [09/1032(0008)/2015-EMR-I]. 9. doi: 10.1098/rspb.1993.0123

4. Anstead GM, Chandrasekar B, Zhao W, Yang J, Perez LE, Melby PC. Malnutrition alters the innate immune response and increases early visceralization following Leishmania donovani infection. Infect Immun. (2001) 69:4709-18. doi: 10.1128/IAI.69.8.47094718.2001 
5. Guillou H, Zadravec D, Martin PG, Jacobsson A. The key roles of elongases and desaturases in mammalian fatty acid metabolism: Insights from transgenic mice. Prog Lipid Res. (2010) 49:186-99. doi: 10.1016/j.plipres.2009.12.002

6. Holman RT, Susan BJ, Osvaldo M, Herminia JI, Maria AR, Maria ET. Essential fatty acid deficiency in malnourished children. Am J Clin Nutr. (1981) 34:1534-9. doi: 10.1093/ajcn/34.8.1534

7. Leichsenring $M$, Ahmed $H$, Laryea MD, Welchering T, Eisa OA, Abbas $\mathrm{OK}$, et al. Polyunsaturated and essential fatty acids in malnourished children. Nutr Res. (1992) 12:595-603. doi: 10.1016/S0271-5317(05) 80029-6

8. Saini S, Kottarath SK, Dinda AK, Dube A, Sahasrabuddhe AA, Thakur CP, et al. Preventive as well as therapeutic significances of linoleic acid in the containment of Leishmania donovani infection. Biochimie. (2020) 175:1322. doi: 10.1016/j.biochi.2020.04.024

9. George $\mathrm{OB}$, Mildred MB. On the nature and role of the fatty acids essential in nutrition. J Biol Chem. (1930) 86:587621. doi: 10.1016/S0021-9258(20)78929-5

10. Hartop PJ, Prottey CJ. Changes in transepidermal water loss and the composition of epidermal lecithin after applications of pure fatty acid triglycerides to skin of essential fatty acid-deficient rats. Br J Dermatol. (1976) 95:255-64. doi: 10.1111/j.1365-2133.1976.tb07012.x

11. Anstead GM, Zhang Q, Melby PC. Malnutrition promotes prostaglandin over leukotriene production and dysregulates eicosanoid-cytokine crosstalk in activated resident macrophages. Prostaglandins Leukot Essent Fatty Acids. (2009) 81:41-51. doi: 10.1016/j.plefa.2009. 04.011

12. Sen E, Chattopadhyay S, Bandopadhyay S, De T, Roy S. Macrophage heterogeneity, antigen presentation, and membrane fluidity: implications in visceral Leishmaniasis. Scand J Immunol. (2001) 53:111-20. doi: 10.1046/j.1365-3083.2001.00856.x

13. Calder PC. Dietary modification of inflammation with lipids. Proc Nutr Soc. (2002) 61:345-58. doi: 10.1079/PNS2002166

14. Violette SH, Ebtisam AH. Synopsis of arachidonic acid metabolism: a review. J Adv Res. (2018) 11:23-32. doi: 10.1016/j.jare.2018.03.005

15. Calder PC, Bond JA, Harvey DJ, Gordon S, Newsholme EA. Uptake and incorporation of saturated and unsaturated fatty acids into macrophage lipids and their effect upon macrophage adhesion and phagocytosis. Biochem J. (1990) 269:807-14. doi: 10.1042/bj2690807

16. Calder PC. Polyunsaturated fatty acids, inflammation, and immunity. Lipids. (2001) 36:1007-24. doi: 10.1007/s11745-001-0812-7

17. Murray H, Xiang Z, Ma X. Responses to Leishmania donovani in mice deficient in both phagocyte oxidase and inducible nitric oxide synthase. Am J Trop Med Hyg. (2006) 74:1013-5. doi: 10.4269/ajtmh.2006.74.1013

18. Jianga WG, Eynardb AR, Mansela RE. The pathology of essential fatty acid deficiency: is it cell adhesion mediated? Med Hypotheses. (2000) 55:25762. doi: 10.1054/mehy.1998.1191

19. Courtney LM, Mattias S, Paul MK. Leishmania-induced inhibition of macrophage antigen presentation analyzed at the single-cell level. J Immunol. (2003) 171:6706-13. doi: 10.4049/jimmunol.171.12.6706

20. Harizi H, Corcuff JB, Gualde N. Arachidonic-acid-derived eicosanoids: roles in biology and immunopathology. Trends Mol Med. 14:4619. doi: 10.1016/j.molmed.2008.08.005

21. Shinomiya S, Naraba H, Ueno A, Utsunomiya I, Maruyama T, Ohuchida S, et al. Regulation of TNF $\alpha$ and interleukin- 10 production by prostaglandins I 2 and E2: studies with prostaglandin receptor-deficient mice and prostaglandin E-receptor subtype-selective synthetic agonists. Biochem Pharmacol. (2001) 61:1153-60. doi: 10.1016/S0006-2952(01)00586-X

22. Cheon H, Rho YH, Choi SJ, Lee YH, Song GG, Sohn J, et al. Prostaglandin E2 augments IL-10 signaling and function. J Immunol. (2006) 177:1092100. doi: 10.4049/jimmunol.177.2.1092

23. Hilkens CM, Snijders A, Snijdewint FG, Wierenga EA, Kapsenberg ML. Modulation of T-cell cytokine secretion by accessory cell-derived products. Eur Respir J (Suppl). (1996) 22:90s-4s.
24. Dennis E, Norris P. Eicosanoid storm in infection and inflammation. Nat Rev Immunol. (2015) 15:511-23. doi: 10.1038/nri3859

25. Saini S, Singh B, Prakash S, Kumari S, Kureel AK, Dube A, et al. Parasitic load determination by differential expressions of 5-lipoxygenase and PGE2 synthases in visceral leishmaniasis. Prostaglandins Other Lipid Mediat. (2019) 147:106390. doi: 10.1016/j.prostaglandins.2019.106390

26. Guimaraes TE, Santos AL, dos Santos RR, Teixeira MM, dos Santos LCW, Soare BPM. Role of interleukin-4 and prostaglandin E2 in Leishmania amazonensis infection of BALB/c mice. Microbes Infect. (2006) 8:121926. doi: 10.1016/j.micinf.2005.11.011

27. Bhattacharjee A, Majumder S, Das S, Ghosh S, Biswas S, Majumdar S. Leishmania donovani-induced prostaglandin E2 Generation is critically dependent on host toll-like receptor 2-cytosolic phospholipase A2 signaling. Infect Immun. (2016) 84:2963-73. doi: 10.1128/IAI.00528-16

28. Saha A, Biswas A, Srivastav S, Mukherjee M, Das PK, Ukil A. Prostaglandin E2 negatively regulates the production of inflammatory cytokines/chemokines and IL-17 in visceral leishmaniasis. J Immunol. (2014) 193:2330-9. doi: 10.4049/jimmunol.1400399

29. França-Costa J, Van Weyenbergh J, Boaventura VS, Luz NF, MaltaSantos H, Souza Oliveira MC, et al. Arginase I, polyamine, and prostaglandin E2 pathways suppress the inflammatory response and contribute to diffuse cutaneous leishmaniasis. J Infect Dis. (2015) 211:426-35. doi: 10.1093/infdis/jiu455

30. Araújo-Santos T, Prates DB, Andrade BB, Nascimento DO, Clarêncio J, Entringer PF, et al. Lutzomyia longipalpis saliva triggers lipid body formation and prostaglandin E2 production in murine macrophages. PLOS Neglect Trop Dis. (2010) 4:e873. doi: 10.1371/journal.pntd.0000873

31. Araújo-Santos T, Prates DB, França-Costa J, Luz NF, Andrade BB, Miranda JC, et al. Prostaglandin E2/Leukotriene B4 balance induced by Lutzomyia longipalpis saliva favors Leishmania infantum infection. Parasit Vectors. (2014) 7:601. doi: 10.1186/s13071-014-0601-8

32. Chaves MM, Marques-da-Silva C, Monteiro AP, Canetti C, CoutinhoSilva R. Leukotriene B4 modulates P2X7 receptor-mediated Leishmania amazonensis elimination in murine macrophages. J Immunol. (2014) 192:4765-73. doi: 10.4049/jimmunol.1301058

33. Morato CI, da Silva IA, Borges AF, Dorta ML, Oliveira MA, Jancar $\mathrm{S}$, et al. Essential role of leukotriene B4 on Leishmania (Viannia) braziliensis killing by human macrophages. Microbes Infect. (2014) 16:94595. doi: 10.1016/j.micinf.2014.08.015

34. Saini S, Dube A, Sahasrabuddhe AA, Thakur CP, Joshi S, Rawat K, et al. Comparison between immuno-clinicopathological features of experimental and human visceral leishmaniasis by Leishmania donovani. Acta Parasitol. (2019) 65:57-67. doi: 10.2478/s11686-019-00127-8

35. Saini S, Rai AK. Hamster, a close model for visceral leishmaniasis: opportunities and Challenges. Parasit Immunol. (2020) 42:e12768. doi: 10.1111/pim.12768

36. Saini S, Rai AK. Linoleic acid inhibits the release of Leishmania donovani derived microvesicles and decreases its survival in macrophages. Front Cell Infect Microbiol. (2020) 10:406. doi: 10.3389/fcimb.2020.00406

37. Nugent AP, Roche HM, Noone EJ, Long A, Kelleher DK, Gibney MJ. The effects of conjugated linoleic acid supplementation on immune function in healthy volunteers. Eur J Clin Nutr. (2005) 59:742-50. doi: 10.1038/sj.ejcn.1602132

Conflict of Interest: The authors declare that the research was conducted in the absence of any commercial or financial relationships that could be construed as a potential conflict of interest.

Copyright (C) 2021 Saini and Rai. This is an open-access article distributed under the terms of the Creative Commons Attribution License (CC BY). The use, distribution or reproduction in other forums is permitted, provided the original author(s) and the copyright owner(s) are credited and that the original publication in this journal is cited, in accordance with accepted academic practice. No use, distribution or reproduction is permitted which does not comply with these terms. 